\title{
Off-pump technique for insertion of a HeartMate vented electric left ventricular assist device
}

\author{
Valentino Piacentino III, BA, Janice Jones, RNP, Carol A. Fisher, BA, \\ Arun K. Singhal, MD, PhD, Mahender Macha, MD, \\ James B. McClurken, MD, and Satoshi Furukawa, MD, \\ Philadelphia, $\mathrm{Pa}$
}

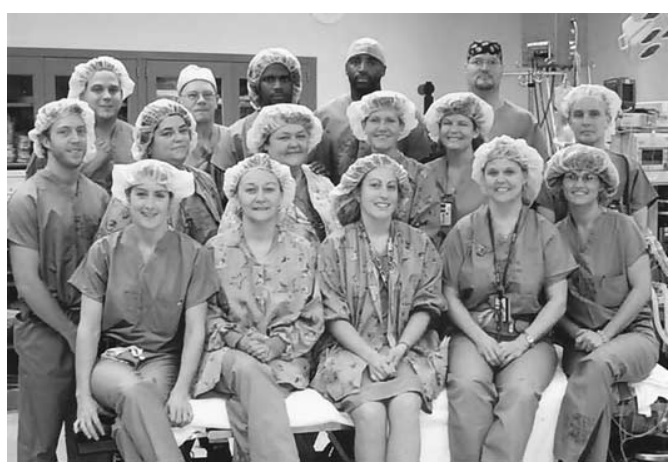

Temple Hospital Cardiac and Thoracic Surgery Team
$\mathrm{T}$ he number of patients awaiting heart transplantation has increased exponentially during the past 10 years, while the number of donor organs has remained unchanged ${ }^{1}$. Alternatives to cardiac transplantation, such as left ventricular assist devices (LVADs), have been shown to decrease mortality and increase the quality of life in patients with end-stage heart failure ${ }^{2}$. It has recently been proposed that these devices may become a destination therapy for patients with end-stage heart failure and thus allow definitive treatment for those who otherwise may not be able to receive or qualify for heart transplantation ${ }^{3}$.

Cardiopulmonary bypass (CPB) is routinely required for implantation of LVADs. However, the well-described complications of CPB may exacerbate multiple organ failure and increase blood product transfusions during and after the operation. Recent technologic advances, including cardiac stabilization devices, have allowed coronary artery bypass surgery to be performed without CPB. We present a technique for insertion without cardiopulmonary bypass of a HeartMate Vented Electric LVAD (Thoratec Corporation, Pleasanton, Calif).

\section{Methods}

Clinical summary. A 60-year-old man was awaiting heart transplantation because of severe end-stage heart failure with dilated cardiomyopathy. The patient had no history of previous cardiac operations or percutaneous coronary angioplasties. Decompensation of his heart failure required transfer to the cardiac intensive care unit and inotropic support with dobutamine (10

From the Division of Cardiac and Thoracic Surgery, Department of Surgery, Temple University Hospital, Philadelphia, $\mathrm{Pa}$.

Received for publication July 23, 2002; accepted for publication Nov 26, 2002.

Address for reprints: Satoshi Furukawa, MD, Chief, Division of Cardiac and Thoracic Surgery, Surgical Director, Cardiopulmonary Transplantation, Department of Surgery, 300 Parkinson Pavilion, 3401 N Broad St, Philadelphia, PA 19140 (E-mail: furukas@tuhs.temple.edu).

J Thorac Cardiovasc Surg 2004;127:262-4

$0022-5223 / \$ 30.00$

Copyright $\odot 2004$ by The American Association for Thoracic Surgery

doi:10.1016/S0022-5223(03)00218-6 $\mu \mathrm{g} /[\mathrm{kg} \cdot \min ])$, milrinone $(0.2 \mu \mathrm{g} /[\mathrm{kg} \cdot \min ])$, and intra-aortic balloon pulsation. His cardiac index was $1.6 \mathrm{~L} /\left(\mathrm{min} \cdot \mathrm{m}^{2}\right)$. Transthoracic echocardiography revealed biventricular dilatation with an ejection fraction of 5\% and a left ventricular end-diastolic dimension of $5.9 \mathrm{~cm}$. Mild-to-moderate mitral regurgitation was evident, and no aortic regurgitation was noted. Because no donor heart was available, the decision was made to place the LVAD.

Operative technique. The patient was transported to the operating room for implantation of a HeartMate Vented Electric LVAD. After general anesthesia was administered and the patient was prepared and draped, a median sternotomy was performed, and the incision was extended to the umbilicus. The pericardium was opened, revealing a large, dilated heart. The dissection was continued in the abdomen for placement of the LVAD body in a preperitoneal position. At this point both pleural cavities were entered, and pleural fluid was evacuated. The diaphragm was then detached anteriorly to allow placement of the apical LVAD inflow cannula in continuation with the preperitoneal space on the left side of the abdomen. At this point the patient was given 40,000 units of heparin sulfate. The activated clotting time was $>1000$ seconds.

An aortic cannula was placed in the ascending aortic root, and a purse-string suture was placed in the right atrium. To stabilize the heart during LVAD inflow cannula placement, the Medtronic Starfish (Medtronic, Minneapolis, Minn) retraction system was deployed. Suction was applied, and the stabilizer pods were placed on the right ventricular apical area lateral to the left anterior descending coronary artery. Deep pericardial sutures were placed ${ }^{4}$. With torsion of the heart toward the right and cephalad, mean arterial and pulmonary pressures were maintained. The HeartMate apical coring knife (Thoratec Laboratories) was used to slightly score the left ventricular apical epicardium, creating a marker for subsequent sewing cuff suture placement. A 2-0 Prolene pursestring suture (Ethicon, Inc, Somerville, NJ) was placed approximately $1 \mathrm{~cm}$ outside of this original coring circle. To secure the apical sewing ring, 9 horizontal mattress sutures (2-0 Ethibond; Ethicon) were placed. These sutures were passed through the apical sewing ring and then sewn to the myocardium, with suture passing just outside of the coring circle to just outside of the purse-string suture. The sutures were pledget supported, and the 
sewing ring was tied down to the myocardium (Figure 1, A). The sewing ring was placed to encompass the left ventricular tissue core that would be removed shortly. Next, the LVAD was brought up to the field, and plastic tie bands were placed around the sewing ring. At this point a 2-0 Prolene suture was placed through the middle of the eventual myocardial tissue core for the LVAD inflow and passed within the coring knife. The coring knife was then placed through the sewing ring, and the left ventricular apex was cored out (Figure 1, B). After one ventricular contraction occurred and blood emerged from the sewing ring, the apical portion of the LVAD body was placed and secured with plastic tie bands and 2 silk ligatures. The $2-0$ purse-string suture previously placed was snared down to completely tighten the coring area and sewing cuff (Figure 1, C). At this point the LVAD was then placed in the preperitoneal space, and the heart was slowly retracted down and placed back in its original position with removal of the stabilization device.

The aortic side of the LVAD body remained closed until outflow cannulation. The aortic conduit was measured, and a side-biting clamp was placed on the ascending aorta. After aortotomy, the LVAD outflow conduit was sewn to the ascending aorta with a running 4-0 Prolene suture with an autologous pericardial pledget. A crossclamp was placed on the outflow conduit near the aortic anastomosis, and the outflow conduit was connected to the assist device. The device was deaired through a primary stab incision in the outflow graft by way of the left ventricular cardiac output, and the LVAD was actuated with the hand pump. Once deairing was completed, the outflow conduit clamp was released, and the LVAD was slowly started. The deairing hole was closed primarily. LVAD flows of 4 to $5 \mathrm{~L} / \mathrm{min}$ were reached. Hemostasis was obtained, and protamine was administered. A pericardial patch was placed under the LVAD body to buttress the peritoneum, and the incision was closed in standard fashion. No blood products were administered during the course of the operation or during the postoperative period.

\section{Discussion}

LVAD insertion can reverse low tissue perfusion and allow rehabilitation of the patient with end-stage heart failure who is awaiting heart transplantation. In addition, LVADs may represent a destination therapy for patients who are not candidates for heart transplantation. $^{3}$

Cardiopulmonary bypass can cause multiple disorders, including immunologic, hepatic, renal, and respiratory dysfunction. ${ }^{5}$ Nonetheless, LVAD placement requires access to and insertion of a cannula into the left ventricular apex. Manipulation of the left ventricular apex can cause hemodynamic compromise and may not be well tolerated in patients with end-stage heart failure. Many patients with end-stage heart failure have multiorgan failure and would therefore be at risk for complications related to the adverse sequelae associated with the blood-synthetic interactions of the CPB circuit. Because off-pump coronary revascularization has been shown to be efficacious in high-risk patients with similar comorbidities, we adapted this surgical technique for LVAD implantation. $^{6}$

To avoid the use of CPB and still allow access to the left ventricular apex, we chose to stabilize the heart with a cardiac stabilization device originally designed for off-pump coronary artery bypass surgery. These devices use suction to fix the heart in


Figure 1. Illustration depicting suture placement for apical sewing cuff for LVAD inflow cannula and cardiac stabilization device on right ventricular free wall (A). With stabilization of heart, coring knife is inserted in sewing cuff (B). Once tissue core is removed, LVAD inflow cannula is placed in left ventricle (C) and secured.

place and allow cephalization of the left ventricular apex without hemodynamic compromise. The Medtronic Starfish is unique as it allows fixation through epicardial surfaces other than the left ventricular apex, including the right ventricular free wall.

It is important to note that our patient did not require blood products. Elimination of cardiopulmonary bypass reduces platelet 
destruction and dysfunction, hemodilution, and resulting coagulopathy. Avoidance of blood products may reduce the elevation in prereactive antibodies commonly observed in patients awaiting heart transplantation with LVADs.

This communication represents the application of off-pump technology for inserting a HeartMate Vented Electric LVAD with a cardiac stabilization device. This technique may reduce the morbidity and mortality associated with LVAD implantation and expand the indications for LVADs to patients with end-stage heart failure. This would be especially beneficial for older high risk patients not considered heart transplant candidates, such as those patients enrolled in the REMATCH trial (Randomized Evaluation of Mechanical Assistance for the Treatment of Congestive Heart Failure). ${ }^{3}$ Furthermore, for those patients who require an intracardiac repair (intraventricular thrombus removal or septal defect repairs) necessitating CPB at the time of the HeartMate Vented Electric LVAD implantation, our technique would permit off-pump preparation of the sewing cuff and aortic outflow cannula, thereby reducing CPB support time and its associated sequelae.

\section{Addendum}

The patient described in the preceding brief communication underwent successful transplantation 278 days ( $\sim 9$ months) after his off-pump HeartMate VE implantation. He is currently recovering at home undergoing rehabilitation.

\section{References}

1. Hosenpud JD, Bennett LE, Keck BM, Boucek MM, Novick RJ. The Registry of the International Society for Heart and Lung Transplantation: eighteenth Official Report-2001. J Heart Lung Transplant. 2001; 20:805-15.

2. Kasirajan V, McCarthy PM, Hoercher KJ, Starling RC, Young JB, Banbury MK, et al. Clinical experience with long-term use of implantable left ventricular assist devices: indications, implantation, and outcomes. Semin Thorac Cardiovasc Surg. 2000;12:229-37.

3. Rose EA, Gelijns AC, Moskowitz AJ, Heitjan DF, Stevenson LW, Dembitsky W, et al. long-term use of a left ventricular assist device for end-stage heart failure. $N$ Engl J Med. 2001;345:1435-43.

4. Bedi HS, Suri A, Kalkat MS, Sengar BS, Mahajan V, Chawla R, et al. Global myocardial revascularization without cardiopulmonary bypass using innovative techniques for myocardial stabilization and perfusion. Ann Thorac Surg. 2000;69:156-64.

5. Hall RI, Smith MS, Rocker G. The systemic inflammatory response to cardiopulmonary bypass: pathophysiological, therapeutic, and pharmacological considerations. Anesth Analg. 1997;805:766-82.

6. Cleveland JC Jr, Shroyer AL, Chen AY, Peterson E, Grover FL. Off-pump coronary artery bypass grafting decreases risk-adjusted mortality and morbidity. Ann Thorac Surg. 2001;72:1282-8.

\title{
Management of aortic insufficiency in patients with left ventricular assist devices: A simple coaptation stitch method (Park's stitch)
}

\author{
Soon J. Park, MD, ${ }^{a}$ Kenneth K. Liao, MD, ${ }^{a}$ Romualdo Segurola, MD, ${ }^{a}$ K. P. Madhu, MD, ${ }^{b}$ and Leslie W. Miller, MD, \\ Minneapolis, Minn
}



eft ventricular assist device (LVAD) implantation increases survival and quality of life for patients with advanced heart failure. ${ }^{1}$ Because of device design, competence of the native aortic valve is critically important. Even trivial aortic insufficiency could worsen with time, ${ }^{2}$ and the

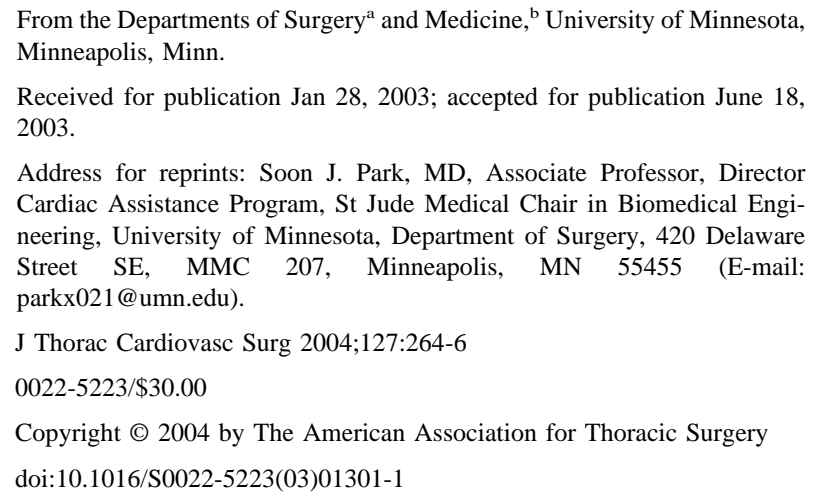

regurgitant flow could make the device ineffective for hemodynamic support.

Managing native aortic valve insufficiency at the time of LVAD implantation has been challenging. Various procedures to prevent aortic insufficiency have been anecdotally reported (eg, aortic valve replacement with a bioprosthetic valve or with a polytetrafluoroethylene patch, or primary closure of the aortic valve opening). ${ }^{3-5}$ Such procedures have been performed successfully, but they could make surgery more complicated. We describe a simple coaptation stitch method of dealing with central aortic insufficiency at the time of LVAD implantation in 2 patients.

\section{Case Summaries}

PATIENT 1. A 61-year-old man with a long-standing history of ischemic cardiomyopathy had a myocardial infarction and was in cardiogenic shock. The LVAD (HeartMate-XVE, Thoratec Corporation, Pleasanton, Calif) was implanted uneventfully. Because of patent venous grafts, anastomosis of the outflow graft to the aorta was completed under aortic crossclamping. Through the aortotomy incision between two proximal vein grafts we were able 